\title{
Nursing students' perception of their clinical practice in intensive care units: A study from Egypt
}

\author{
Nahed Attia Kandeel, Hanaa Hussein Ahmed \\ Critical Care and Emergency Nursing Department, Mansoura University, Egypt
}

Received: January 31, 2019

Accepted: March 19, 2019

Online Published: April 9, 2019

DOI: $10.5430 /$ jnep.v9n7p101

URL: https://doi.org/10.5430/jnep.v9n7p101

\begin{abstract}
Providing nursing students with quality clinical experience in intensive care units (ICUs) is a major concern for nursing educators in Egypt. Understanding nursing students' perception of their critical care experience is important in future planning of successful clinical placements in ICUs. The purpose of this study was to investigate undergraduate nursing students' perception of their clinical practice in ICUs. The study involved 306 nursing students who were registered in critical care nursing course. Data were collected using a self-administered survey which addressed nursing students' perception of three domains including clinical practice environment, clinical teaching and learning and factors hindering clinical practice in intensive care setting. The results illustrated that the majority of students enjoyed their clinical experience in ICUs. However, students highlighted many factors that hindered their clinical practice such as the stressful intensive care setting, fear of making mistakes, complex patients' conditions, theory-practice gap, overburdening with documentation and lack of coordination between clinical placements. Supportive learning environment is needed to enhance students' clinical learning, improve collaboration between students, demonstrators and critical care nursing staff, and reduce theory-practice gap.
\end{abstract}

Key Words: Clinical practice, Intensive care units, Nursing students, Perception

\section{INTRODUCTION}

The goal of undergraduate nursing education is to prepare qualified nurses who are committed to provide high quality, safe patient care. ${ }^{[1]}$ Nursing is a practice discipline with clinical experience being an integral component of nursing education. ${ }^{[2]}$ Clinical practice allows nursing students to develop competencies required for professional practice and transform theoretical knowledge into patient care. ${ }^{[3]}$ It meets accreditation requirements, supports students' skill acquisition, ${ }^{[4]}$ and ensures the ability of nursing student to practice as a nurse.$^{[5]}$ Hence, the quality of clinical preparation of nursing students has a great influence on the quality of the program.
Critical care placement offers a wealth of learning opportunities for nursing students. ${ }^{[6]}$ It allows students to practice important technical skills, communication skills and health teaching skills, and to observe health care team collaborative work. ${ }^{[7]}$ Clinical practice in ICUs exposes students to a variety of complex critical care conditions and nursing procedures which broaden their knowledge and enhance their clinical competence. ${ }^{[6]}$ A study conducted by Gallagher, Rice, Tierney, Page and McKinney ${ }^{[8]}$ to evaluate a 2-day critical care course showed that nursing students' knowledge, skills and confidence had been improved by the end of the course. It has been also reported that despite the challenges facing nursing students during critical care placement, students' self-confidence had improved after critical care

\footnotetext{
*Correspondence: Nahed Attia Kandeel; Email: Nahed_Kandeel2000@yahoo.com; Address: Critical Care and Emergency Nursing Department, Mansoura University, Egypt.
} 
experience. ${ }^{[9,10]}$

Providing nursing students with quality clinical experience within a supportive clinical learning environment is a major concern for academic institutions. ${ }^{[11]}$ Therefore, understanding nursing students' perception of their critical care experience is important in future planning of successful clinical placement. Studies which investigated this area are scarce, especially in Egypt.

\subsection{Context of the study}

The Bachelor of nursing program in Egypt is a hospital-based program more than fifty years ago. It is a 4-year program, followed by one internship year. After graduation, the students are qualified to work as registered nurses. Clinical practice is the heart of undergraduate nursing education. Within the Bachelor of nursing program at Faculty of Nursing, Mansoura University, students study critical care nursing course in level four. This course aims to help students to acquire knowledge and skills required for providing safe, competent nursing care for critically ill patients in different intensive care settings. Clinical practice in ICUs is a requirement for completing this course. Hence, students receive hands-on experience across nine clinical placements including Medical ICU, Surgical ICU, Neuro ICU, General ICU, Hepatic ICU, Gastrointestinal ICU, Anesthesia ICU, Coronary Care Unit and Recovery ICU. The clinical placement lasts 13 weeks. The students spend 6 hours/week in the clinical placement, and two hours training per week in the skill lab. These placements provide nursing students the opportunity to integrate knowledge and nursing skills into practice. The traditional model for clinical supervision is adopted. Demonstrators from critical care and emergency nursing department at Faculty of Nursing are assigned to support and supervise nursing students during clinical practice. The teacher-student ratio is nearly 1 to 12 . Preceptorship model of clinical education has not yet been adopted in undergraduate clinical education in the Faculty. However, critical care nurses sometimes allow students to participate in caring activities of critically ill patients under their supervision. At the end of each semester, students' feedback about the course is collected and used as a base for enhancing the course in the following semester. However, the evaluation is general with no specific focus on clinical practice which is the core component of the course. Enhancing students' clinical experience in ICUs requires academic staff and demonstrators to get access to students' perception of their clinical practice.

\subsection{Aim}

The aim of this study was to investigate undergraduate nursing students' perception of their clinical practice in ICUs of 102
Mansoura University Hospital, Egypt.

\section{METHOD}

\subsection{Design}

A survey design was used to collect data concerning nursing students' perception of their clinical practice in ICUs.

\subsection{Participants}

A convenience sample of fourth level nursing students who were enrolled in critical care nursing course in the first semester of the academic year 2017-2018 $(n=416)$ was included in the study.

\subsection{Setting}

The study was conducted at Faculty of Nursing, Mansoura University which is one of the biggest universities in Egypt. Faculty of Nursing is accredited by the National Authority for Quality Assurance and Accreditation. It receives nearly between 400 and 600 students each year. The Faculty offers programs designed to meet the needs of health care institutions including Bachelor of Nursing, Masters in Nursing and Doctoral programs. The Faculty involves eight departments; one of them is critical care and emergency nursing department which is responsible for teaching critical care nursing course for level four nursing students.

\subsection{Ethical considerations}

Ethical approval was granted from the Research Ethics Committee of Faculty of Nursing, Mansoura University (ref. 159/2017). Additional approval was obtained from the Dean of Faculty of Nursing. A cover letter including the aim of the study, the voluntariness nature of participation and other elements of informed consent was included with the survey sheet. Completion of the survey and returning it back was indicative of students' informed consent. In order to maintain anonymity and confidentiality of the participants, completed questionnaires were collected back anonymously.

\subsection{Instrument}

Data were collected using a self-administered survey which was developed by the researchers based upon relevant literature. ${ }^{[11-13]}$ It consists of three main domains. The first domain was comprised of 8 statements inquiring about clinical practice environment. The second domain incorporated 12 statements assessing clinical teaching and learning as perceived by nursing students. The third domain included 10 statements addressing factors hindering students' clinical practice in ICUs. Participants were asked to record their responses to items on a five-point Likert scale ( 1 = "strongly disagree", 2 = "disagree", 3 = "uncertain", 4 = "agree" and 5 $=$ "strongly agree"). Content validity of the instrument was 
determined by 5 experts from critical care and emergency nursing department. The instrument has been shown to be highly reliable with a Cronbach's alpha of 0.829. A pilot study was conducted on 42 nursing students to check the clarity and applicability of the instrument. Modifications were made accordingly. Participants of the pilot study were excluded from the study sample.

\subsection{Data collection}

Students were informed about the study after they had completed their clinical placement during the last lecture of the critical care nursing course. The questionnaire was distributed to all available students $(n=342)$ who were given the opportunity to answer the questionnaire in the lecture theater. Students spent from 15 to 20 minutes completing the survey. The questionnaire were collected back in the lecture theater.

\subsection{Data analysis}

Data were analyzed using The Statistical Package for Social Science version 21.0 (SPSS, Chicago, IL, USA). Quantitative data were expressed as median and interquartile range (IQR). Qualitative data were compared by one sample ChiSquare test. The non-parametric alternative Mann-Whitney U-test was used to compare quantitative data. Statistically significance level was set at $p \leq .05$.

\section{Results}

A total of 306 nursing students completed the questionnaire reflecting an overall response rate of $89.5 \%$. Of these, 63 were excluded as their questionnaire had over $20 \%$ missing data, leaving 243 for data analysis (see Figure 1). All participants were between the age of 19 and 21 years old, and $85.2 \%$ were females and $11.5 \%$ were males. Eight students $(3.3 \%)$ did not declare their gender.

Table 1 shows nursing students' perception of clinical practice environment in ICUs. Most of the students agreed that clinical placement in ICUs was suitable for achieving their clinical practice goals $(73.2 \%)$, exposed them to a variety of critical conditions and caring activities (84.8\%) and was a good learning environment $(77 \%)$. Despite the fact that the majority of the students $(76.5 \%)$ enjoyed their clinical experience in ICUs, they perceived it as a stressful place for clinical practice $(86.8 \%)$.

Table 2 depicts nursing students' perception of clinical teaching and learning in ICUs. The majority of students were satisfied with the quality of clinical teaching and learning ( $p$ $<.0005)$. They agreed that clinical practice in ICUs provided them the opportunity to participate in morning patient care $(95.5 \%)$ and variety of caring activities (85.2\%), develop their clinical skills $(79.4 \%)$ and build confidence in caring Published by Sciedu Press for critically ill patients $(69.1 \%)$. However, $54.4 \%$ felt that the focus was on documentation rather than their learning needs, and $44.9 \%$ highlighted a gap between theory and practice. Moreover, $32.1 \%$ did not receive any feedback about their performance.



Figure 1. Flow chart of the study participants

Table 3 illustrates nursing students' perception of factors hindering clinical practice in ICUs. For most students, factors hindered their clinical practice were the stressful nature of intensive care setting (91.4\%), fear of making mistakes $(86.4 \%)$, the complexity of patients' conditions (86\%), gap between theory and clinical practice $(72.4 \%)$, focusing on documentation rather than clinical performance (72\%) and lack of coordination between clinical placements $(67.9 \%)$. For nearly half of the students, lack of critical care staff's support (57.2\%), inadequate clinical hours (46.5\%) and lack of feedback about clinical performance affected their clinical practice negatively (48.1\%). Moreover, 37.4\% reported inadequate supervision in clinical setting. 
Table 1. Nursing students' perceptions of clinical practice environment

\begin{tabular}{|c|c|c|c|c|c|c|c|c|}
\hline \multirow[b]{2}{*}{ Statements } & \multicolumn{5}{|c|}{ Students' Perception $(n=243)(\%)$} & \multicolumn{2}{|c|}{ Total count $(\%)$ of } & \multirow[b]{2}{*}{$p$} \\
\hline & $\begin{array}{l}\text { Strongly } \\
\text { Disagree }\end{array}$ & Disagree & Uncertain & Agree & $\begin{array}{l}\text { Strongly } \\
\text { Agree }\end{array}$ & Agree & Disagree & \\
\hline $\begin{array}{l}\text { Clinical placement was suitable for } \\
\text { achieving my clinical practice goals. }\end{array}$ & $\begin{array}{l}1 \\
(0.4 \%)\end{array}$ & $\begin{array}{l}23 \\
(9.5 \%)\end{array}$ & $\begin{array}{l}41 \\
(16.9 \%)\end{array}$ & $\begin{array}{l}134 \\
(55.1 \%)\end{array}$ & $\begin{array}{l}44 \\
(18.1 \%)\end{array}$ & $\begin{array}{l}178 \\
(73.2 \%)\end{array}$ & $\begin{array}{l}24 \\
(9.9 \%)\end{array}$ & $<.0005$ \\
\hline $\begin{array}{l}\text { Clinical placement in different ICUs } \\
\text { exposed me to a variety of critical } \\
\text { conditions and caring activities. }\end{array}$ & $\begin{array}{l}2 \\
(0.8 \%)\end{array}$ & $\begin{array}{l}8 \\
(3.3 \%)\end{array}$ & $\begin{array}{l}27 \\
(11.1 \%)\end{array}$ & $\begin{array}{l}117 \\
(48.1 \%)\end{array}$ & $\begin{array}{l}89 \\
(36.6 \%)\end{array}$ & $\begin{array}{l}206 \\
(84.8 \%)\end{array}$ & $\begin{array}{l}10 \\
(4.1 \%)\end{array}$ & $<.0005$ \\
\hline $\begin{array}{l}\text { Critical care staffs were cooperative } \\
\text { and supportive. }\end{array}$ & $\begin{array}{l}16 \\
(6.6 \%)\end{array}$ & $\begin{array}{l}67 \\
(27.6 \%)\end{array}$ & $\begin{array}{l}56 \\
(23.0 \%)\end{array}$ & $\begin{array}{l}88 \\
(36.2 \%)\end{array}$ & $\begin{array}{l}16 \\
(6.6 \%)\end{array}$ & $\begin{array}{l}104 \\
(42.8 \%)\end{array}$ & $\begin{array}{l}83 \\
(34.2 \%)\end{array}$ & .025 \\
\hline $\begin{array}{l}\text { Good communication with } \\
\text { demonstrators and critical care nurses } \\
\text { improved my clinical experience. }\end{array}$ & $\begin{array}{l}10 \\
(4.1 \%)\end{array}$ & $\begin{array}{l}67 \\
(27.6 \%)\end{array}$ & $\begin{array}{l}49 \\
(20.2 \%)\end{array}$ & $\begin{array}{l}86 \\
(35.4 \%)\end{array}$ & $\begin{array}{l}31 \\
(12.8 \%)\end{array}$ & $\begin{array}{l}117 \\
(48.1 \%)\end{array}$ & $\begin{array}{l}77 \\
(31.7 \%)\end{array}$ & .564 \\
\hline $\begin{array}{l}\text { The clinical hours were adequate to } \\
\text { accomplish the assigned tasks. }\end{array}$ & $\begin{array}{l}11 \\
(4.5 \%)\end{array}$ & $\begin{array}{l}56 \\
(23.0 \%)\end{array}$ & $\begin{array}{l}49 \\
(20.2 \%)\end{array}$ & $\begin{array}{l}91 \\
(37.4 \%)\end{array}$ & $\begin{array}{l}36 \\
(14.8 \%)\end{array}$ & $\begin{array}{l}127 \\
(52.3 \%)\end{array}$ & $\begin{array}{l}67 \\
(27.5 \%)\end{array}$ & .480 \\
\hline $\begin{array}{l}\text { Intensive care setting was a good } \\
\text { learning environment. }\end{array}$ & $\begin{array}{l}1 \\
(0.4 \%)\end{array}$ & $\begin{array}{l}18 \\
(7.4 \%)\end{array}$ & $\begin{array}{l}37 \\
(15.2 \%)\end{array}$ & $\begin{array}{l}112 \\
(46.1 \%)\end{array}$ & $\begin{array}{l}75 \\
(30.9 \%)\end{array}$ & $\begin{array}{l}187 \\
(77 \%)\end{array}$ & $\begin{array}{l}19 \\
(7.8 \%)\end{array}$ & $<.0005$ \\
\hline $\begin{array}{l}\text { Intensive care setting was stressful } \\
\text { place for clinical practice. }\end{array}$ & $\begin{array}{l}3 \\
(1.2 \%)\end{array}$ & $\begin{array}{l}13 \\
(5.3 \%)\end{array}$ & $\begin{array}{l}16 \\
(6.6 \%)\end{array}$ & $\begin{array}{l}91 \\
(37.4 \%)\end{array}$ & $\begin{array}{l}120 \\
(49.4 \%)\end{array}$ & $\begin{array}{l}211 \\
(86.8 \%)\end{array}$ & $\begin{array}{l}16 \\
(6.6 \%)\end{array}$ & $<.0005$ \\
\hline $\begin{array}{l}\text { I enjoyed my clinical experience in } \\
\text { ICUs. }\end{array}$ & $\begin{array}{l}7 \\
(2.9 \%)\end{array}$ & $\begin{array}{l}22 \\
(9.1 \%)\end{array}$ & $\begin{array}{l}28 \\
(11.5 \%)\end{array}$ & $\begin{array}{l}97 \\
(39.9 \%)\end{array}$ & $\begin{array}{l}89 \\
(36.6 \%)\end{array}$ & $\begin{array}{l}186 \\
(76.5 \%)\end{array}$ & $\begin{array}{l}29 \\
(12 \%)\end{array}$ & $<.0005$ \\
\hline
\end{tabular}

Note. Data are presented as count and percentage; $p$ value is presented by one-sample chi-square test. $p$ value $\leq .05$.

Table 2. Nursing students' perception of clinical teaching and learning in ICUs

\begin{tabular}{|c|c|c|c|c|c|c|c|c|}
\hline \multirow[b]{2}{*}{ Statements } & \multicolumn{5}{|c|}{ Students' Perception $(n=243)(\%)$} & \multicolumn{2}{|c|}{ Total count (\%) of } & \multirow[b]{2}{*}{$p$} \\
\hline & $\begin{array}{l}\text { Strongly } \\
\text { Disagree }\end{array}$ & Disagree & Uncertain & Agree & $\begin{array}{l}\text { Strongly } \\
\text { Agree }\end{array}$ & Agree & Disagree & \\
\hline $\begin{array}{l}\text { The goal of clinical practice was clear } \\
\text { to me. }\end{array}$ & $\begin{array}{l}1 \\
(0.4 \%)\end{array}$ & $\begin{array}{l}9 \\
(3.7 \%)\end{array}$ & $\begin{array}{l}43 \\
(17.7 \%)\end{array}$ & $\begin{array}{l}121 \\
(49.8 \%)\end{array}$ & $\begin{array}{l}69 \\
(28.4 \%)\end{array}$ & $\begin{array}{l}190 \\
(78.2 \%)\end{array}$ & $\begin{array}{l}10 \\
(4.1 \%)\end{array}$ & $<.0005$ \\
\hline $\begin{array}{l}\text { I was fully aware of the required } \\
\text { competencies for passing the course. }\end{array}$ & $\begin{array}{l}1 \\
(0.4 \%)\end{array}$ & $\begin{array}{l}14 \\
(5.8 \%)\end{array}$ & $\begin{array}{l}41 \\
(16.9 \%)\end{array}$ & $\begin{array}{l}112 \\
(46.1 \%)\end{array}$ & $\begin{array}{l}75 \\
(30.9 \%)\end{array}$ & $\begin{array}{l}187 \\
(77 \%)\end{array}$ & $\begin{array}{l}15 \\
(6.1 \%)\end{array}$ & $<.0005$ \\
\hline $\begin{array}{l}\text { The demonstrator was always } \\
\text { available in the clinical placement. }\end{array}$ & $\begin{array}{l}8 \\
(3.3 \%)\end{array}$ & $\begin{array}{l}19 \\
(7.8 \%)\end{array}$ & $\begin{array}{l}14 \\
(5.8 \%)\end{array}$ & $\begin{array}{l}103 \\
(42.4 \%)\end{array}$ & $\begin{array}{l}99 \\
(40.7 \%)\end{array}$ & $\begin{array}{l}202 \\
(83.1 \%)\end{array}$ & $\begin{array}{l}27 \\
(11.1 \%)\end{array}$ & $<.0005$ \\
\hline $\begin{array}{l}\text { The demonstrator guided me } \\
\text { whenever I needed. }\end{array}$ & $\begin{array}{l}8 \\
(3.3 \%)\end{array}$ & $\begin{array}{l}10 \\
(4.1 \%)\end{array}$ & $\begin{array}{l}27 \\
(11.1 \%)\end{array}$ & $\begin{array}{l}110 \\
(45.3 \%)\end{array}$ & $\begin{array}{l}88 \\
(36.2 \%)\end{array}$ & $\begin{array}{l}198 \\
(81.5 \%)\end{array}$ & $\begin{array}{l}18 \\
(7.4 \%)\end{array}$ & $<.0005$ \\
\hline $\begin{array}{l}\text { Bed-side teaching helped me to } \\
\text { develop my clinical skills. }\end{array}$ & $\begin{array}{l}4 \\
(1.6 \%)\end{array}$ & $\begin{array}{l}9 \\
(3.7 \%)\end{array}$ & $\begin{array}{l}37 \\
(15.2 \%)\end{array}$ & $\begin{array}{l}111 \\
(45.7 \%)\end{array}$ & $\begin{array}{l}82 \\
(33.7 \%)\end{array}$ & $\begin{array}{l}193 \\
(79.4 \%)\end{array}$ & $\begin{array}{l}13 \\
(5.3 \%)\end{array}$ & $<.0005$ \\
\hline $\begin{array}{l}\text { I participated in morning routine } \\
\text { patient care with critical care nurses. }\end{array}$ & $\begin{array}{l}0.0 \\
(0.0 \%)\end{array}$ & $\begin{array}{l}7 \\
(2.9 \%)\end{array}$ & $\begin{array}{l}4 \\
(1.6 \%)\end{array}$ & $\begin{array}{l}75 \\
(30.9 \%)\end{array}$ & $\begin{array}{l}157 \\
(64.6 \%)\end{array}$ & $\begin{array}{l}232 \\
(95.5 \%)\end{array}$ & $\begin{array}{l}7 \\
(2.9 \%)\end{array}$ & $<.0005$ \\
\hline $\begin{array}{l}\text { I had the opportunity to participate in } \\
\text { a variety of caring activities under } \\
\text { supervision. }\end{array}$ & $\begin{array}{l}2 \\
(0.8 \%)\end{array}$ & $\begin{array}{l}16 \\
(6.6 \%)\end{array}$ & $\begin{array}{l}18 \\
(7.4 \%)\end{array}$ & $\begin{array}{l}142 \\
(58.4 \%)\end{array}$ & $\begin{array}{l}65 \\
(26.7 \%)\end{array}$ & $\begin{array}{l}207 \\
(85.2 \%)\end{array}$ & $\begin{array}{l}18 \\
(7.4 \%)\end{array}$ & $<.0005$ \\
\hline $\begin{array}{l}\text { The focus of clinical practice was my } \\
\text { learning needs rather than } \\
\text { documentation. }\end{array}$ & $\begin{array}{l}48 \\
(19.8 \%)\end{array}$ & $\begin{array}{l}84 \\
(34.6 \%)\end{array}$ & $\begin{array}{l}53 \\
(21.8 \%)\end{array}$ & $\begin{array}{l}42 \\
(17.3 \%)\end{array}$ & $\begin{array}{l}16 \\
(6.6 \%)\end{array}$ & $\begin{array}{l}58 \\
(23.9 \%)\end{array}$ & $\begin{array}{l}132 \\
(54.4 \%)\end{array}$ & $<.0005$ \\
\hline $\begin{array}{l}\text { What I learned in classroom and skill } \\
\text { lab was what I saw in clinical } \\
\text { practice. }\end{array}$ & $\begin{array}{l}41 \\
(16.9 \%)\end{array}$ & $\begin{array}{l}68 \\
(28.0 \%)\end{array}$ & $\begin{array}{l}42 \\
(17.3 \%)\end{array}$ & $\begin{array}{l}72 \\
(29.6 \%)\end{array}$ & $\begin{array}{l}20 \\
(8.2 \%)\end{array}$ & $\begin{array}{l}92 \\
(37.9 \%)\end{array}$ & $\begin{array}{l}109 \\
(44.9 \%)\end{array}$ & $<.0005$ \\
\hline $\begin{array}{l}\text { I was able to integrate my knowledge } \\
\text { into practice. }\end{array}$ & $\begin{array}{l}7 \\
(2.9 \%)\end{array}$ & $\begin{array}{l}30 \\
(12.3 \%)\end{array}$ & $\begin{array}{l}52 \\
(21.4 \%)\end{array}$ & $\begin{array}{l}120 \\
(49.4 \%)\end{array}$ & $\begin{array}{l}34 \\
(14.0 \%)\end{array}$ & $\begin{array}{l}154 \\
(63.4 \%)\end{array}$ & $\begin{array}{l}37 \\
(15.2 \%)\end{array}$ & $<.0005$ \\
\hline $\begin{array}{l}\text { Hands-on training helped me to build } \\
\text { confidence in my clinical skills. }\end{array}$ & $\begin{array}{l}5 \\
(2.1 \%)\end{array}$ & $\begin{array}{l}18 \\
(7.4 \%)\end{array}$ & $\begin{array}{l}52 \\
(21.4 \%)\end{array}$ & $\begin{array}{l}114 \\
(46.9 \%)\end{array}$ & $\begin{array}{l}54 \\
(22.2 \%)\end{array}$ & $\begin{array}{l}168 \\
(69.1 \%)\end{array}$ & $\begin{array}{l}23 \\
(9.5 \%)\end{array}$ & $<.0005$ \\
\hline $\begin{array}{l}\text { The demonstrator provided me with } \\
\text { feedback about my clinical } \\
\text { performance continuously. }\end{array}$ & $\begin{array}{l}32 \\
(13.2 \%)\end{array}$ & $\begin{array}{l}46 \\
(18.9 \%)\end{array}$ & $\begin{array}{l}43 \\
(17.7 \%)\end{array}$ & $\begin{array}{l}89 \\
(36.6 \%)\end{array}$ & $\begin{array}{l}33 \\
(13.6 \%)\end{array}$ & $\begin{array}{l}122 \\
(50.2 \%)\end{array}$ & $\begin{array}{l}78 \\
(32.1 \%)\end{array}$ & .949 \\
\hline
\end{tabular}

Note. Data are presented as count and percentage; $p$ value is presented by one-sample chi-square test. $p$ value $\leq .05$. 
Table 3. Nursing students' perceptions of factors hindering clinical practice in ICUs

\begin{tabular}{|c|c|c|c|c|c|c|c|c|}
\hline \multirow[b]{2}{*}{ Factors } & \multicolumn{5}{|c|}{ Students' Perception (n = 243) (\%) } & \multicolumn{2}{|c|}{ Total count (\%) of } & \multirow[b]{2}{*}{$p$} \\
\hline & $\begin{array}{l}\text { Strongly } \\
\text { Disagree }\end{array}$ & Disagree & Uncertain & Agree & $\begin{array}{l}\text { Strongly } \\
\text { Agree }\end{array}$ & Agree & Disagree & \\
\hline $\begin{array}{l}\text { The complexity of patients' } \\
\text { conditions. }\end{array}$ & $\begin{array}{l}6 \\
(2.5 \%)\end{array}$ & $\begin{array}{l}16 \\
(6.6 \%)\end{array}$ & $\begin{array}{l}12 \\
(4.9 \%)\end{array}$ & $\begin{array}{l}77 \\
(31.7 \%)\end{array}$ & $\begin{array}{l}132 \\
(54.3 \%)\end{array}$ & $\begin{array}{l}209 \\
(86 \%)\end{array}$ & $\begin{array}{l}22 \\
(9.1 \%)\end{array}$ & $<.0005$ \\
\hline $\begin{array}{l}\text { The stressful nature of intensive } \\
\text { care setting. }\end{array}$ & $\begin{array}{l}0.0 \\
(0.0 \%)\end{array}$ & $\begin{array}{l}13 \\
(5.3 \%)\end{array}$ & $\begin{array}{l}8 \\
(3.3 \%)\end{array}$ & $\begin{array}{l}94 \\
(38.7 \%)\end{array}$ & $\begin{array}{l}128 \\
(52.7 \%)\end{array}$ & $\begin{array}{l}222 \\
(91.4 \%)\end{array}$ & $\begin{array}{l}13 \\
(5.3 \%)\end{array}$ & $<.0005$ \\
\hline $\begin{array}{l}\text { Fear of making mistakes when } \\
\text { caring for critically ill patients. }\end{array}$ & $\begin{array}{l}4 \\
(1.6 \%)\end{array}$ & $\begin{array}{l}16 \\
(6.6 \%)\end{array}$ & $\begin{array}{l}13 \\
(5.3 \%)\end{array}$ & $\begin{array}{l}69 \\
(28.4 \%)\end{array}$ & $\begin{array}{l}141 \\
(58.0 \%)\end{array}$ & $\begin{array}{l}210 \\
(86.4 \%)\end{array}$ & $\begin{array}{l}20 \\
(8.2 \%)\end{array}$ & $<.0005$ \\
\hline $\begin{array}{l}\text { Gap between what was learned } \\
\text { in classroom and real clinical } \\
\text { practice. }\end{array}$ & $\begin{array}{l}3 \\
(1.2 \%)\end{array}$ & $\begin{array}{l}27 \\
(11.1 \%)\end{array}$ & $\begin{array}{l}37 \\
(15.2 \%)\end{array}$ & $\begin{array}{l}75 \\
(30.9 \%)\end{array}$ & $\begin{array}{l}101 \\
(41.6 \%)\end{array}$ & $\begin{array}{l}176 \\
(72.4 \%)\end{array}$ & $\begin{array}{l}30 \\
(12.3 \%)\end{array}$ & $<.0005$ \\
\hline $\begin{array}{l}\text { Inadequate supervision in } \\
\text { clinical setting. }\end{array}$ & $\begin{array}{l}23 \\
(9.5 \%)\end{array}$ & $\begin{array}{l}96 \\
(39.5 \%)\end{array}$ & $\begin{array}{l}33 \\
(13.6 \%)\end{array}$ & $\begin{array}{l}56 \\
(23.0 \%)\end{array}$ & $\begin{array}{l}35 \\
(14.4 \%)\end{array}$ & $\begin{array}{l}91 \\
(37.4 \%)\end{array}$ & $\begin{array}{l}119 \\
(49 \%)\end{array}$ & $<.0005$ \\
\hline $\begin{array}{l}\text { Lack of support of critical care } \\
\text { nurses. }\end{array}$ & $\begin{array}{l}6 \\
(2.5 \%)\end{array}$ & $\begin{array}{l}52 \\
(21.4 \%)\end{array}$ & $\begin{array}{l}46 \\
(18.9 \%)\end{array}$ & $\begin{array}{l}92 \\
(37.9 \%)\end{array}$ & $\begin{array}{l}47 \\
(19.3 \%)\end{array}$ & $\begin{array}{l}139 \\
(57.2 \%)\end{array}$ & $\begin{array}{l}58 \\
(23.9 \%)\end{array}$ & .025 \\
\hline $\begin{array}{l}\text { Inadequate clinical practice } \\
\text { hours. }\end{array}$ & $\begin{array}{l}33 \\
(13.6 \%)\end{array}$ & $\begin{array}{l}67 \\
(27.6 \%)\end{array}$ & $\begin{array}{l}30 \\
(12.3 \%)\end{array}$ & $\begin{array}{l}58 \\
(23.9 \%)\end{array}$ & $\begin{array}{l}55 \\
(22.6 \%)\end{array}$ & $\begin{array}{l}113 \\
(46.5 \%)\end{array}$ & $\begin{array}{l}100 \\
(41.2 \%)\end{array}$ & .275 \\
\hline $\begin{array}{l}\text { Lack of feedback about clinical } \\
\text { performance. }\end{array}$ & $\begin{array}{l}19 \\
(7.8 \%)\end{array}$ & $\begin{array}{l}59 \\
(24.3 \%)\end{array}$ & $\begin{array}{l}48 \\
(19.8 \%)\end{array}$ & $\begin{array}{l}59 \\
(24.3 \%)\end{array}$ & $\begin{array}{l}58 \\
(23.9 \%)\end{array}$ & $\begin{array}{l}117 \\
(48.1 \%)\end{array}$ & $\begin{array}{l}78 \\
(32.1 \%)\end{array}$ & .564 \\
\hline $\begin{array}{l}\text { Focusing on documentation } \\
\text { rather than clinical } \\
\text { performance. }\end{array}$ & $\begin{array}{l}11 \\
(4.5 \%)\end{array}$ & $\begin{array}{l}35 \\
(14.4 \%)\end{array}$ & $\begin{array}{l}22 \\
(9.1 \%)\end{array}$ & $\begin{array}{l}66 \\
(27.2 \%)\end{array}$ & $\begin{array}{l}109 \\
(44.9 \%)\end{array}$ & $\begin{array}{l}175 \\
(72 \%)\end{array}$ & $\begin{array}{l}46 \\
(18.9 \%)\end{array}$ & $<.0005$ \\
\hline $\begin{array}{l}\text { Lack of coordination between } \\
\text { clinical placements. }\end{array}$ & $\begin{array}{l}7 \\
(2.9 \%)\end{array}$ & $\begin{array}{l}38 \\
(15.6 \%)\end{array}$ & $\begin{array}{l}33 \\
(13.6 \%)\end{array}$ & $\begin{array}{l}75 \\
(30.9 \%)\end{array}$ & $\begin{array}{l}90 \\
(37.0 \%)\end{array}$ & $\begin{array}{l}165 \\
(67.9 \%)\end{array}$ & $\begin{array}{l}45 \\
(18.5 \%)\end{array}$ & $<.0005$ \\
\hline
\end{tabular}

Note. Data are presented as count and percentage; $p$ value is presented by one-sample chi-square test. $p$ value $\leq .05$.

Table 4 shows gender difference in perception of clinical practice in ICUs. No statistical significant differences were found between male and female students regarding their perception of the three domains.
It was noted that a considerable percentage of students had uncertainty about aspects of clinical practice environment, clinical teaching and learning, and factors hindered their clinical practice in ICUs.

Table 4. Gender difference in perception of clinical practice in ICUs

\begin{tabular}{llll}
\hline \multirow{2}{*}{ Domain } & \multicolumn{1}{l}{ Gender $(\mathbf{n}=\mathbf{2 3 5})$} & \multirow{2}{*}{$\boldsymbol{p}$ value } \\
\cline { 2 - 4 } & Male $(\mathbf{n}=\mathbf{2 8})$ & Female $(\mathbf{n}=\mathbf{2 0 7})$ & .874 \\
\hline Percent CPE score & $75(70-81.9)$ & $75(67.5-82.5)$ & .183 \\
Percent CTL score & $78.3(73.8-81.7)$ & $75(68.3-83.3)$ & .345 \\
Percent FHCP score & $72(68-80)$ & $76(68-84)$ & .449 \\
Percent Grand Total score & $75.7(72.3-79.8)$ & $74.7(70.7-79.5)$ & .49
\end{tabular}

Note. CPE: Clinical Practice Environment; CTL: Clinical Teaching and Learning; FHCP: Factors Hindering Clinical Practice; $p$ value $\leq .05 ;$ Data are presented as Median and Interquartile Range (IQR) and compared by Mann-Whitney U-test.

\section{DiscuSSION}

The current study investigated nursing students' perception of their clinical practice in ICUs. The findings suggested that the majority of students enjoyed their clinical experience in ICUs and perceived it as a good learning opportunity. However, they viewed the intensive care setting as a stressful place for clinical practice. These findings are consistent Published by Sciedu Press with other studies describing the experience of undergraduate nursing students in ICUs. ${ }^{[10,14]}$ The stressful nature of ICU was also perceived by the vast majority of students as a factor that hindered their clinical practice. This is consistent with other previous studies which highlighted the benefits of critical care experience for nursing students but raised some concerns regarding the stressful nature of caring in the ICU, 
and how this could affect students' clinical learning experience. ${ }^{[6,15,16]}$ Critical care setting is a complex environment due to caring for critically ill and unstable patients, dealing with advanced technology, performing invasive procedures and sustaining patients' lives. ${ }^{[17]}$ Therefore, most of the students agreed that fear of making mistakes and the complexity of patients' conditions were factors that hindered their clinical practice in ICUs. This is in line with a Turkish study which reported that students were afraid of causing patients harm during caring in the ICU. ${ }^{[10]}$ This finding suggests that critical care placement for nursing students is very challenging, and requires effective support system to reduce students' fear and anxiety, and enhance their clinical experience. ${ }^{[6]}$

In the current study, a large number of students felt that critical care nurses were neither cooperative nor supportive, and that communication was not effective with demonstrators or critical care nurses. Additionally, more than half of the students believed that lack of critical care staff's support negatively affected their clinical practice. The literature highlighted the challenges facing staff nurses when caring for patients and supervising students simultaneously. One of the challenges is the lack of time allocated for precepting because of nurses' heavy workload. ${ }^{[18]}$ To deal with this challenge, Swinny and Brady ${ }^{[7]}$ suggested involving nursing students in basic patient care activities which will decrease nurses' workload and allow more time for students' clinical teaching and supervision. In the current study, each demonstrator was responsible for supervising from 10 to 12 students. Despite the availability of the demonstrator in one unit, the clinical teaching time for each student was nearly from 20 to 30 minutes which was inadequate to teach students complex procedures and supervise them during providing patient care. These findings are supported by Hansen et al. ${ }^{[19]}$ who emphasized the need for enough time for mentorship to allow students to integrate new knowledge, skills and attitude in clinical practice. In the current study, we suggest reducing clinical instructors-students ratios in the ICU to 1:6 as recommended by Kelly's ${ }^{[20]}$ study as a strategy for enhancing students' communication with their mentors and improving clinical supervision. The importance of mentor-student relationship in enhancing nursing competencies, creating supportive learning environment and increasing students' satisfaction with their clinical placement was reported in previous studies. ${ }^{[1,2,9,11,21]}$

Although clinical placement in the ICU allowed students to participate in patients' caring activities, and helped them to develop their clinical skills, more than half of the students felt that the focus of the practice was on documentation rather than on meeting their learning needs. Moreover, most students viewed focusing on documentation as a hindrance for clinical practice. This could be the reason that nearly a quarter of the students felt that the clinical practice hours were inadequate. Documentation is an integral part of staff nurses' work in all health care settings. It is a mean of communicating information about patients' status, and their response to treatment and care. ${ }^{[22]}$ Hence, accurate documentation is a vital element for safe, quality nursing practice. ${ }^{[23]}$ Despite the fact that nursing students feel burdened with the task of documentation, it is an essential skill that they should develop during their clinical placement. A study conducted in Egypt to assess nursing students' perception of safety of critically ill patients reported that $55 \%$ of nursing students had poor perception of documentation. The investigators related this finding to the limited training time and students being occupied with patients' care. ${ }^{[2]}$ As the time allocation for the clinical training is limited, it should be utilized effectively. ${ }^{[25]}$

In accordance with other studies, the students reported a gap between what they taught in classroom and skill lab, and what they experienced in clinical setting. ${ }^{[26-29]}$ Most students agreed that theory-practice gap affected their clinical training. Theory-practice gap in nursing education has long been an area of concern for teachers and learners, ${ }^{[28]}$ and initiatives to bridge this gap remain under scrutiny. A study conducted by Saifan et al. ${ }^{[27]}$ reported many reasons for theory-practice gap in nursing education. These involve lack of qualifications of the clinical instructors, lack of communication between theory and clinical teachers, poor communication between clinical instructors and students, not considering student individual learning needs, and inappropriate instructor-student ratio. However, this study has a small sample size which limits the generalizability of the findings, but it delivers an overview of the potential reasons of theory-gap practice from nursing students' perspectives. In the current study, we suggest introducing preceptorship model for undergraduate nursing students' critical care practice. Clinical preceptors can be useful in enhancing students' clinical guidance and supervision, facilitating the integration of theoretical knowledge into practice and improving collaboration between clinical placements. ${ }^{[2]}$

The current study illustrated that nearly one third of the students did not receive feedback about their clinical performance and this was perceived as a hindrance for clinical practice. This is consistent with the findings of Shalaby, et al. ${ }^{[24]}$ In other previous studies, nursing students reported the feedback of clinical teachers as an important attribute for successful clinical practice. ${ }^{[20,30]}$ 


\section{Conclusion}

The results of the current study provide a rounded picture of nursing students' perception of their clinical practice in intensive care setting which can be used as a base for updating and enhancing clinical teaching of critical care nursing course. In general, most students enjoyed their clinical practice in ICUs, however, they faced many challenges which negatively affected their clinical experience. Intensive care setting provides nursing students with a variety of learning opportunities. Hence, to ensure high quality clinical practice in ICUs, it is important to provide students with supportive learning environment that focuses on students' learning needs and enhances collaboration between students, clinical instructors and critical care nursing staff. Preceptorship model for clinical education should be adopted to ensure effective clinical learning and supervision. Clinical educators should understand the importance of providing feedback to students about their clinical performance and utilizing the clinical hours effectively. Further qualitative research is needed to explore nursing students' experience of clinical practice in ICUs.

\section{Limitations of the study}

The participants were nursing students from one Faculty of Nursing in Egypt which may hinder the generalizability of the findings.

\section{ACKNOWLEDGeMENTS}

The authors would like to acknowledge Faculty of Nursing, Mansoura University for making this study possible. We would also like to thank nursing students who participated in the study and shared their perception of critical care experience with us.

\section{Conflicts of InTEREST Disclosure}

No conflict of interest has been declared by the authors.

\section{REFERENCES}

[1] Dimitriadou M, Papastavrou E, Efstathiou G, et al. Baccalaureate nursing students' perceptions of learning and supervision in the clinical environment. Nursing and Health Sciences. 2015; 17: 236-242. PMid:25377993 https://doi.org/10.1111/nhs.12174

[2] Phuma-Ngaiyaye E, Bvumbwe T, Chipeta MC. Using preceptors to improve nursing students' clinical learning outcomes: A Malawian students' perspective. International Journal of Nursing Sciences. 2017 Apr; 4(2): 164-168. https://doi.org/10.1016/j.ijnss . 2017 .03 .001

[3] Newton JM, Jolly BC, Ockerby CM, et al. Clinical Learning Environment Inventory: factor analysis. Journal of Advanced Nursing. 2010 Jun; 66(6): 1371-1381.

[4] Lea E, Marlow A, Altmann E, et al. Nursing students' preferences for clinical placements in the residential aged care setting. J Clin Nurs. 2018 Jan; 27(1-2): 143-152.

[5] Bjørk I, Berntsen K, Brynildsen G, et al. Nursing students' perceptions of their clinical learning environment in placements outside traditional hospital settings. Journal of Clinical Nursing. 2014 Oct; 23(19-20): 2958-2967.

[6] Williams E, Palmer C. Student nurses in critical care: benefits and challenges of critical care as a learning environment for student nurses. Nurs Crit Care. 2014 Nov; 19(6): 310-5. PMid:24131570 https://doi.org/10.1111/nicc.12053

[7] Swinny B, Brady M. The benefits and challenges of providing nursing student clinical rotations in the intensive care unit. Crit Care Nurs Q. 2010 Jan-Mar; 33(1): 60-66. PMid:20019511 https : //doi.org/10.1097/CNQ.0b013e3181c8df7c

[8] Gallagher PJ, Rice B, Tierney P, et al. An evaluation of a critical care course for undergraduate nursing students. Nursing in Critical Care. 2011 Aug; 16 (5): 261-269.

[9] Alasad JA, Ahmad MM, Abu Tabar N, et al. Nursing student's experiences in critical care course: a qualitative study. J. Intensive \& Crit Care. 2015 Oct; 1: 1.

Published by Sciedu Press
[10] Tastan S, Lyigun E, Ayhan H, et al. Experiences of Turkish undergraduate nursing students in the intensive care unit. Collegian. 2015; 22 117-123. https://doi.org/10.1016/j.colegn.2013.12.003

[11] Papastavrou E, Dimitriadou M, Tsangari H, et al. Nursing students' satisfaction of the clinical learning environment: a research study. BMC Nursing. 2016 Jul; 15: 44.

[12] Chan D. Development of the clinical learning environment inventory: Using the theoretical framework of learning environment studies to assess nursing students' perceptions of the hospital as a learning environment. The Journal of Nursing Education. 2002 Feb; 41(2): 69-75. PMid:11852986

[13] Saarikoski M, Isoaho H, Warne T, et al. The nurse teacher in clinical practice: developing the new sub-dimension to the clinical learning environment and supervision (CLES) Scale. International Journal of Nursing Studies. 2008 Aug; 45 (8): 1233-1237.

[14] Vatansever N, Akansel N. Intensive care unit experience of nursing students during their clinical placements: A qualitative study. International Journal of Caring Sciences. 2016 Sep-Dec; 9(3): 1040-1048.

[15] Moscaritolo LM. Interventional strategies to decrease nursing student anxiety in the clinical learning environment. Journal of Nursing Education. 2009; 48(1): 17-23. https://doi.org/10.3928/014848 34-20090101-08

[16] Rattray J. In the firing line: the nurse academic in practice. Nursing in Critical Care. 2004 May; 9(3): 95-97. PMid:15152750 https://doi.org/10.1111/j.1362-1017.2004.00068.x

[17] Backes MT, Erdmann AL, Büscher A. The living, dynamic and complex environment care in intensive care unit. Revista LatinoAmericana de Enfermagem. 2015; 23(3): 411-418. PMid:26155009 https://doi.org/10.1590/0104-1169.0568.2570

[18] Carlson E, Pilhammar EA, Wann-Hansson C. Time to precept: supportive and limiting conditions for preceptoring nurses. Journal of Advanced Nursing. 2010; 66(2): 432-441. PMid:20423426 https://doi.org/10.1111/j.1365-2648.2009.05174.x 
[19] Hansen BS, Gundersen EM, Bj $\varnothing \mathrm{rn}^{\circ}$ a GB. Improving student supervision in a Norwegian intensive care unit: a qualitative study. Nursing and Health Sciences. 2011 May; 13: 255-261. PMid:21615657

[20] Kelly C. Student's perception of effective clinical teaching revisited. Nurse Education Today. 2007 Nov; 27(8): 885-892. PMid:17321013 https://doi.org/10.1016/j.nedt.2006.12.005

[21] Jokelainen M, Turunen H, Tossavainen K. A systematic review of mentoring nursing students in clinical placements. Journal of Clinical Nursing. 2011 Mar; 20 (19-20): 2854-2867. PMid:21429055 https://doi.org/10.1111/j.1365-2702.2010.03571.x

[22] Day L. What is documentation for? American Journal of Critical Care. 2009 Jan; 18(1): 77-80. PMid:19116409 https : //doi .org/ $10.4037 /$ ajcc2009419

[23] American Nurses Association. Principles for nursing documentation: guidance for registered nurses. ANA, Silver Spring, Maryland. 2010.

[24] Shalaby SA, Seweid MM, El-soussi AH. Critically ill patient safety in nursing education: Students' practices and perception. International Journal of Healthcare. 2018; 4(2).

[25] IP WY, Chan DS. Hong Kong nursing students' perception of the clinical environment: a questionnaire survey. International Journal of Nursing Studies. 2005 Aug; 42 (6): 665-672. PMid:15978595 https://doi.org/10.1016/j.ijnurstu.2004.09.019

[26] Mahmoud M. Practical learning and theory-practice gap as perceived by nursing students. International Journal of Current Research. 2014; 6(02): 5083-5093.

[27] Saifan A, AbuRuz M, Masa'deh, R. Theory practice gaps in nursing education: A qualitative perspective. Journal of Social Sciences. 2015; 11(1): 20-29. https ://doi.org/10.3844/jssp. 2015.20 .29

[28] Sharif F, Masoumi S. A qualitative study of nursing student experiences of clinical practice. BMC Nursing. $2005 \mathrm{NOV}$; 4 . PMid:16280087 https ://doi.org/10.1186/1472-6955-4-6

[29] Tiwaken SU, Caranto LC, David JJ. The real world: lived experiences of student nurses during clinical practice. International Journal of Nursing Science. 2015; 5(2): 66-75.

[30] Sweet L, Broadbent J. Nursing students' perceptions of the qualities of a clinical facilitator that enhance learning. Nurse Education in Practice. 2017 Jan; 22: 30-36. PMid:27918904 https: //doi.org/10.1016/j.nepr.2016.11.007 